\title{
Article
}

\section{Online Monitoring of Torpedo Car Shells Based on Equal An- gle Scanning}

\author{
Yumei TANG $1,2^{*}$, Kefu LIU 1
}

\author{
1. School of Information Science and Technology, Fudan University, No.220 Handan Road, Shanghai, \\ 200433, P.R. China \\ 2.Hefei GoodTech Co., Ltd., Anhui, Hefei, 230088, goodtek0551@163.com
}

\begin{abstract}
Infrared sensors are applied more and more widely in industrial production applications. Based on the theory of thermal radiation, this paper discusses the system design principle, temperature calibration method and thermal image analysis method in detail. The system has passed the measurement unit certification, showing that the field of view is $180^{\circ}$, the number of scanning points is 2048 , the linear velocity is $10-100 \mathrm{~Hz}$, the spatial resolution is $2.5 \mathrm{mrad}$, and the precision is $\pm 1^{\circ} \mathrm{C}$. On-line monitoring test has been done in the steelmaking plant of Bao Steel. The results show that the system has strong anti-interference ability, stability and reliability, and meets the application requirements of online monitoring.
\end{abstract}

Keywords: Infrared sensors; calibration model; Online monitoring; OCIS codes: (120.280) Remote sensing and sensors; (280.4991) Passive remote sensing

\section{INTRODUCTION}

The torpedo car is a very important large-scale molten iron transportation equipment in the steel industry. It is a torpedo-type molten iron tank used in a large blast furnace. It is also called a mixed iron car. It has good insulation conditions, small heat loss, and insulation time. What's more, it is energy-saving and enduring. Because of the large volume of the car, it can also store molten iron to coordinate the temporary imbalance of ironmaking and steelmaking. At the same time, it can replace the steel-making mixer and ordinary molten iron car to complete desulfurization, dephosphorization and other operations during the transportation of molten iron [1,2].

The lining may crack, bulge and spall due to chemical erosion, mechanical erosion and rapid cooling and heating. Due to its high cost of maintenance, repair and replacement, continuous monitoring and temperature detection are required to determine the lining of the torpedo car. When the refractory material is locally damaged severely but not found, it will cause a serious accident $[3,4]$.

Infrared temperature measurement[5] is an effective, fast and noncontact measurement technology that is especially suitable for measuring the temperature of moving objects[6] .To our knowledge, the traditional measurement method is commonly used, that is the relevant staff carry portable infrared instruments to detect the quantity of torpedo cars on the transportation railway [7]. Not only this method is labor intensive, but also the measurement results are not stable, especially in hot or rainy and snowy weather. In order to improve the efficiency of monitoring and measurement stability, and effectively reduce the labor intensity of related operators, on line infrared sensors are used for scanning and imaging $[8,9]$.

In this work, temperature calibration model is proposed, and an on-line monitoring system for temperature field of torpedo car is developed. The panoramic thermal image of torpedo car is obtained by equiangular scanning. The inner lining thickness is simulated by thermal map, and the temperature map is drawn according to the body shell size. Finally, the safety alarm and accurate positioning are realized. 


\section{THEORY \& METHOD}

\subsection{Infrared radiation theory}

According to Plank's law of black body radiation [10], As long as the temperature of any object in nature is higher than absolute zero $\left(-273.15^{\circ} \mathrm{C}\right)$, it will emit energy in the form of electromagnetic radiation in a very wide range of wavelengths, generating electromagnetic waves (radiation energy). Different materials, different temperatures, different surface brightness, different colors, etc., emit different infrared radiation intensity. Planck's radiation law gives the specific spectral distribution of blackbody radiation. At a certain temperature, the energy radiated by a blackbody per unit area in unit time, unit solid angle and unit wavelength interval is:

$$
\mathrm{B}(\lambda, T)=\frac{2 h c^{2}}{\lambda^{5}} \cdot \frac{1}{\exp \left(\frac{h c}{\lambda K T}\right)-1}
$$

$B(\lambda, T)$ - Blackbody's spectral radiance $\left(W \cdot m^{-2} \cdot S_{r}^{-1} \cdot \mu m^{-1}\right)$

$c$ - Speed of light $\left(2.998 * 10^{8} \mathrm{~m} \cdot \mathrm{s}^{-1}\right)$

$T$ - Absolute temperature of blackbody $(K, T=t+273)$

$h$ - Planck constant $\left(6.626 * 10^{-34} \mathrm{~J} \cdot \mathrm{s}\right)$

$K$ - Boltzmann constant $\left(1.380 * 10^{-23} \mathrm{~J} \cdot \mathrm{K}^{-1}\right)$

At a certain temperature, the spectral radiance of a black body has an extreme value, and the position of this extreme value is related to the temperature. This is the Wien displacement law (Wien)[11]

$$
\lambda_{\mathrm{m}} \times \mathrm{T}=2898(\mu \mathrm{m} \cdot \mathrm{K})
$$

$\lambda_{m}$ : Wavelength at maximum blackbody spectral radiance

$T$ - Absolute temperature of blackbody $(K, T=t+273)$

The normal temperature range of the shell of torpedo car is $100^{\circ} \mathrm{C} \sim 500^{\circ} \mathrm{C}$, so we choose medium wave infrared detector $(3 \sim 5 \mu \mathrm{m})$, probing to get the maximum signal-tonoise ratio.

According to Planck's formula (1), we put forward the voltage response model of the infrared detector (3):

$$
U=\frac{\mathrm{A}}{\exp \left(\frac{B}{\mathrm{t}+273.2}\right)-1}-C
$$

Here, $\mathrm{t}$ is the target radiation temperature, $\mathrm{U}$ is the voltage signal output by the detector, A,B,C is correlation coefficient.

\subsection{System design}

As shown in Fig. 1, we design a high-speed line scan infrared temperature measurement system, which uses synchronous motor scanning and real-time image processing to realize the reconstruction and visualization of the two-dimensional temperature field of the moving target. It is composed of a refrigerated infrared temperature measurement module and a DC brushless Motor synchronous scanning module, high-speed signal acquisition and processing module, computer real-time image splicing and other technical components. The infrared sensor mainly receives the radiant energy emitted by the measured object, focuses it on the detector through the receiving lens, completes the conversion of the optical signal to the electrical signal, and then obtains the voltage value by low noise amplifier, filtering, and analog-to-digital conversion. 


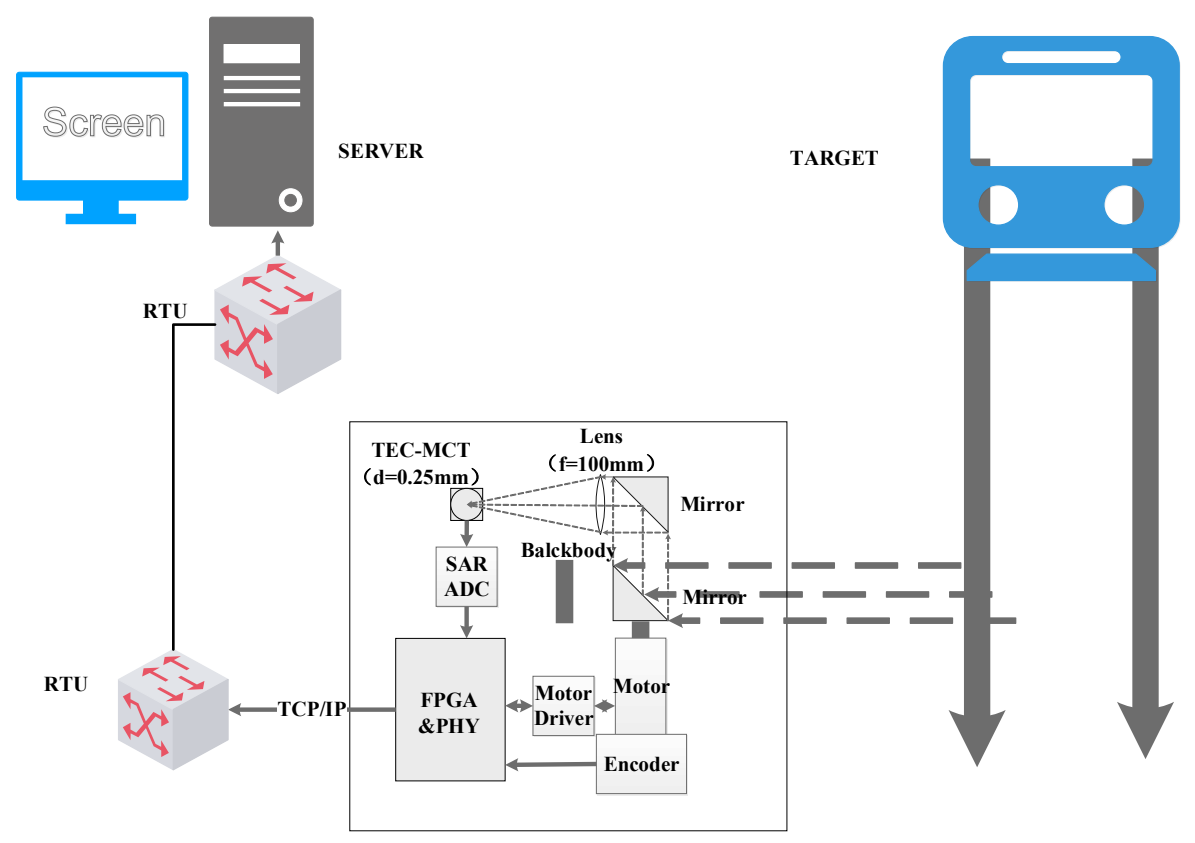

Figure 1. System Block.

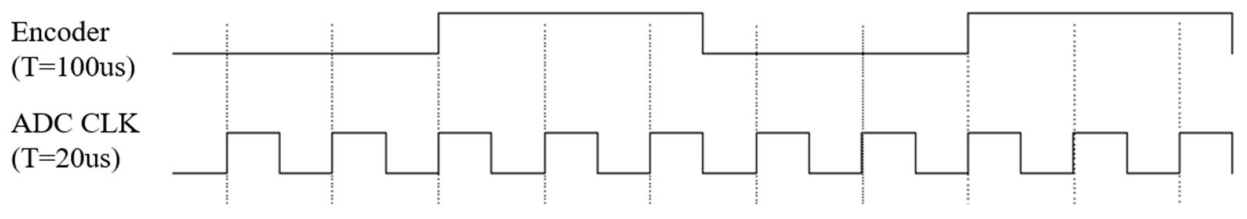

Figure 2. ADC sample based on equal angle scanning.

In Fig.2, the SAR ADC sampling module obtains the voltage signal of the infrared detector $[-5 \mathrm{~V},+5 \mathrm{~V}]$ and converts it into absolute voltage in the range of $[0,5]$.

$$
V_{o}=\frac{V_{\text {in }}+5}{2}-V_{\text {ref }}=a d c_{-} \text {data }-V_{\text {ref }}, \text { Vin } \in[-5,+5]
$$

Here, Vo is the digital output voltage, Vin is the AD sampling output voltage, and Vref is the reference voltage of ADC. 


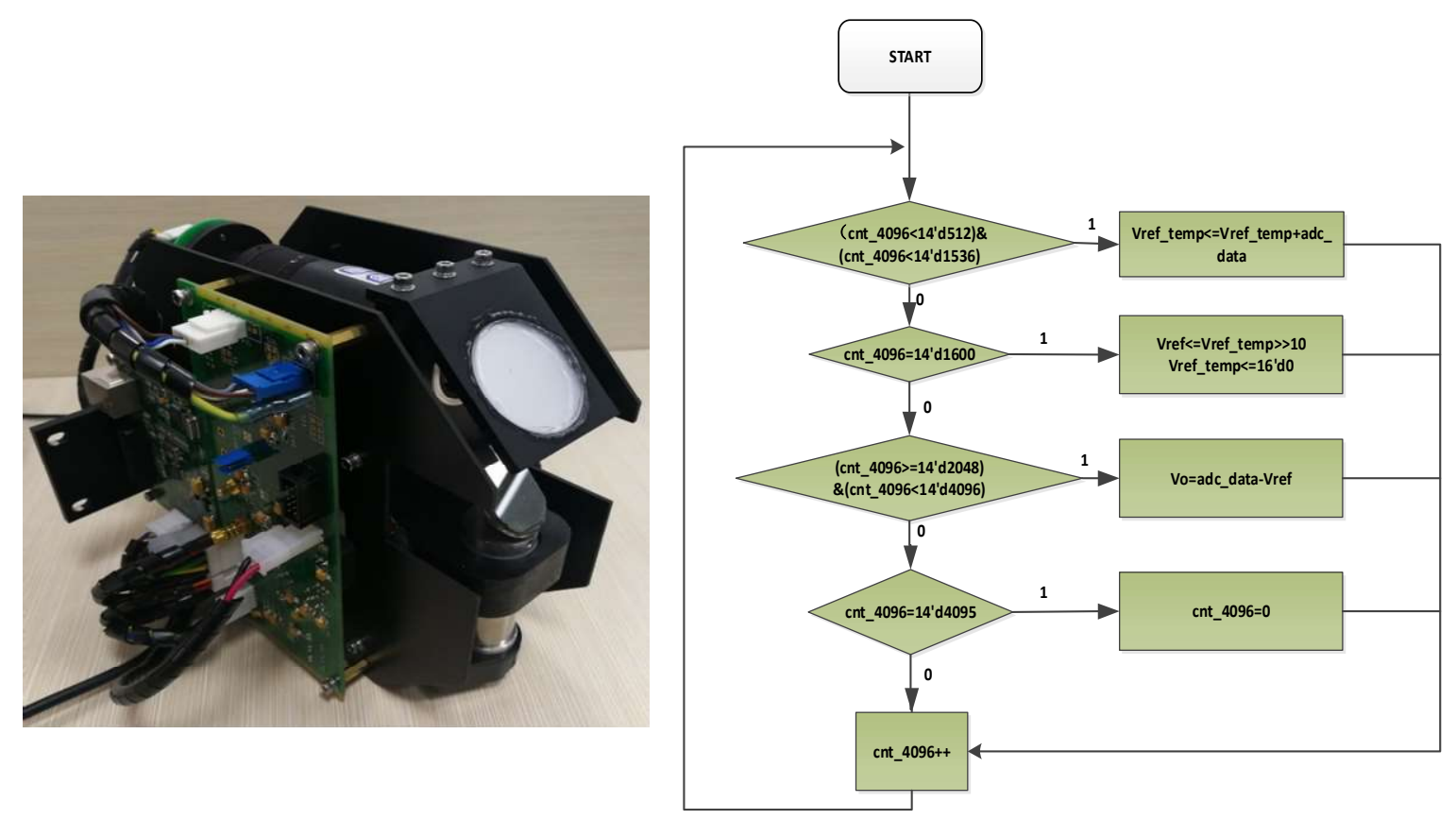

Figure 3. FPGA based digital signal processing with inner blackbody compensation.

As shown in Fig. 3, in order to compensate for the influence of background radiation, we carry out real-time temperature correction through background sampling. The key technical index is listed in table 1.

Table 1. key technical index.

\begin{tabular}{cc}
\hline parameter & value \\
\hline wavelength range & $3-5 \mu \mathrm{m}$ \\
FOV & $180^{\circ}$ \\
focal length & $100 \mathrm{~mm}$ \\
pixel number/line & 2048 \\
sampling rate & $500 \mathrm{kSPS}$ \\
scan speed & $10-100 \mathrm{~Hz}$ \\
precison & $\pm 1^{\circ} \mathrm{C}$ \\
spatial resolution & $2.5 \mathrm{mrad}$ \\
\hline
\end{tabular}

\subsection{Temperature calibration}

As shown in Table 2, we use a standard black body (M315-HT, LumaSense, USA) to calibrate the measurement data of the infrared sensor, and obtain the relationship data between the black body temperature $t$ and the output voltage $U$ of the infrared sensor.

Table 2. calibration data set by blackbody.

\begin{tabular}{cc}
\hline $\mathrm{t}\left({ }^{\circ} \mathrm{C}\right)$ & $\mathrm{U}(\mathrm{V})$ \\
\hline 80 & 0.04 \\
95 & 0.05 \\
100 & 0.06
\end{tabular}




$\begin{array}{lc}130 & 0.12 \\ 150 & 0.18 \\ 200 & 0.42 \\ 230 & 0.65 \\ 250 & 0.85 \\ 260 & 0.95 \\ 270 & 1.2 \\ 280 & 1.3 \\ 290 & 1.4 \\ 300 & 1.55 \\ 310 & 1.7 \\ 320 & 1.9 \\ 330 & 2.1 \\ 340 & 2.3 \\ 350 & 2.5 \\ 380 & 3.25 \\ 400 & 3.8 \\ 450 & 5.45 \\ & \end{array}$

According to formula (3), we can get the temperature inversion formula (4) of the temperature measurement system:

$$
t=\frac{B}{\log \left(\frac{A}{U+C}+1\right)}-273.2
$$

As shown in Fig. 4, we use the Matlab R2016a curve fitting tool to perform curve fitting on formula (3), and obtain the correlation coefficient: $A=631.2, B=3444$, and $\mathrm{C}=0.002758$.

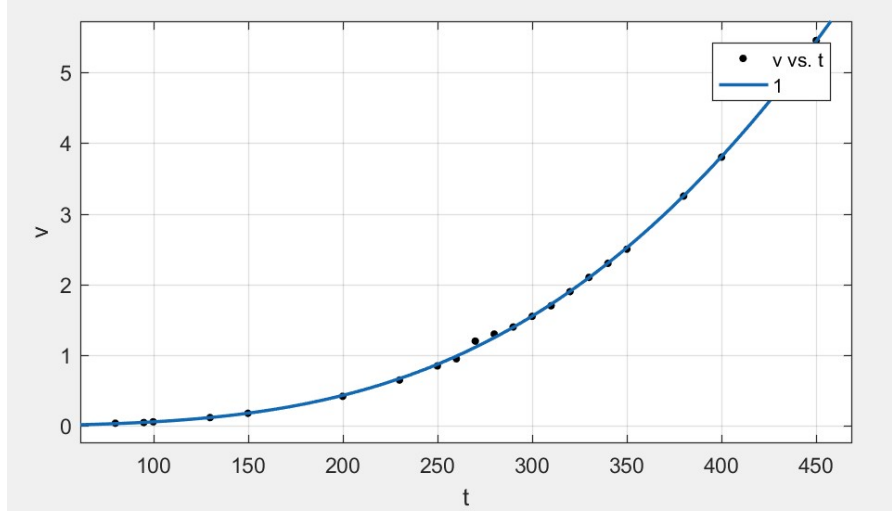

Figure 4. Curve fitting of temperature voltage response model.

\section{EXPERIMENTS}

As shown in Fig.5, the main equipment at the monitoring site includes 2 Scanners (in the red dashed line ellipse in Fig. 5); 4 RFID antennas (in the yellow dashed line circle in Figure 3); and RFID tags installed on the torpedo car (Each torpedo car is equipped with 4 RFID tags, 2 tags both on the front and back sides). The system collects, calculates and 
transmits temperature data on the shell surface, and transmits it to the central control room through an optical fiber network. The software adopts the B/S(Browser/Server) architecture. The main equipment in the central control room is the temperature data server and the large-screen display platform in the console. The large-screen display platform is used for real-time display, and the temperature data server can be used for off-line data analysis.

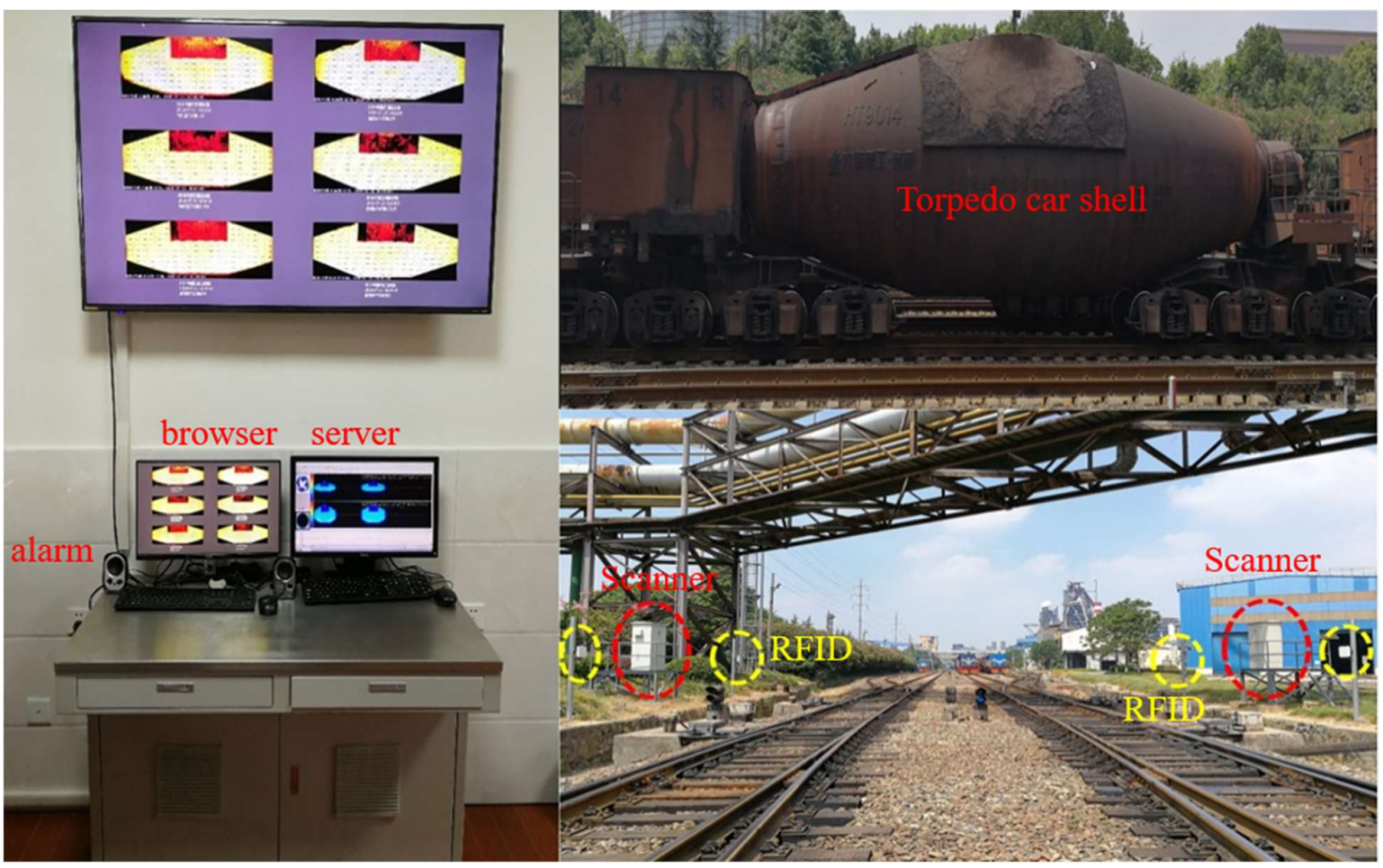

Figure 5. On line monitoring application.

\section{RESULTS AND DISCUSSION}

\subsection{Real time analysis of heat map}

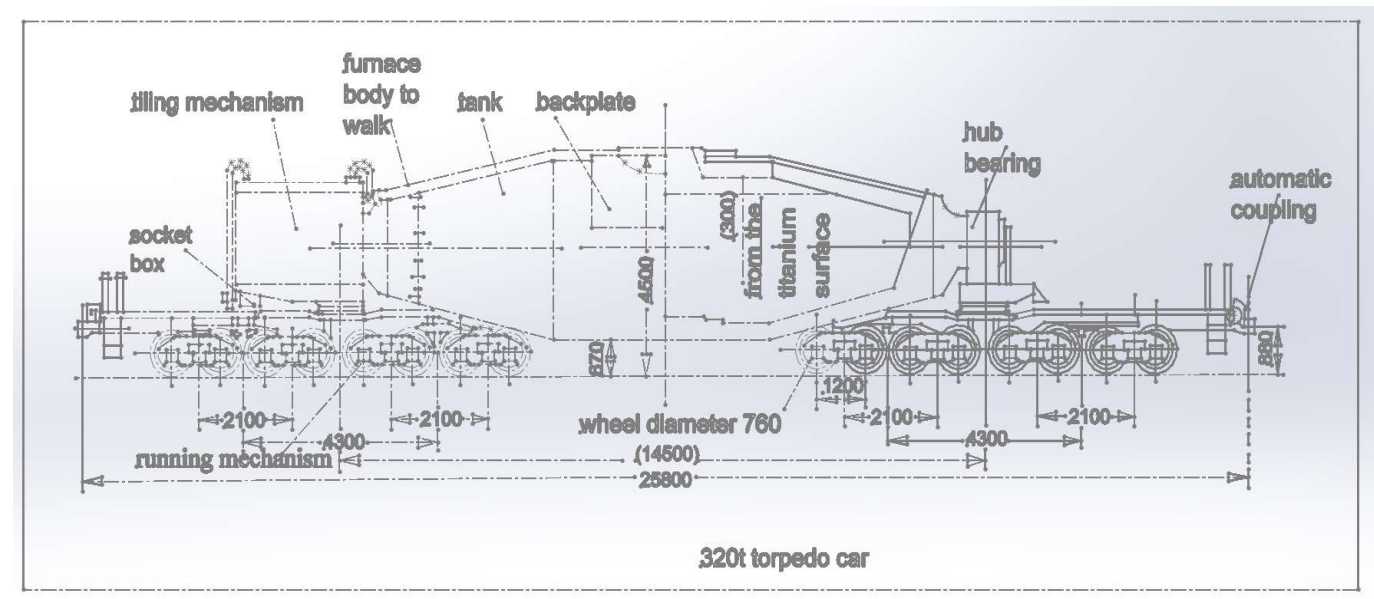

Figure 6. Mechanical of 320t torpedo car. 


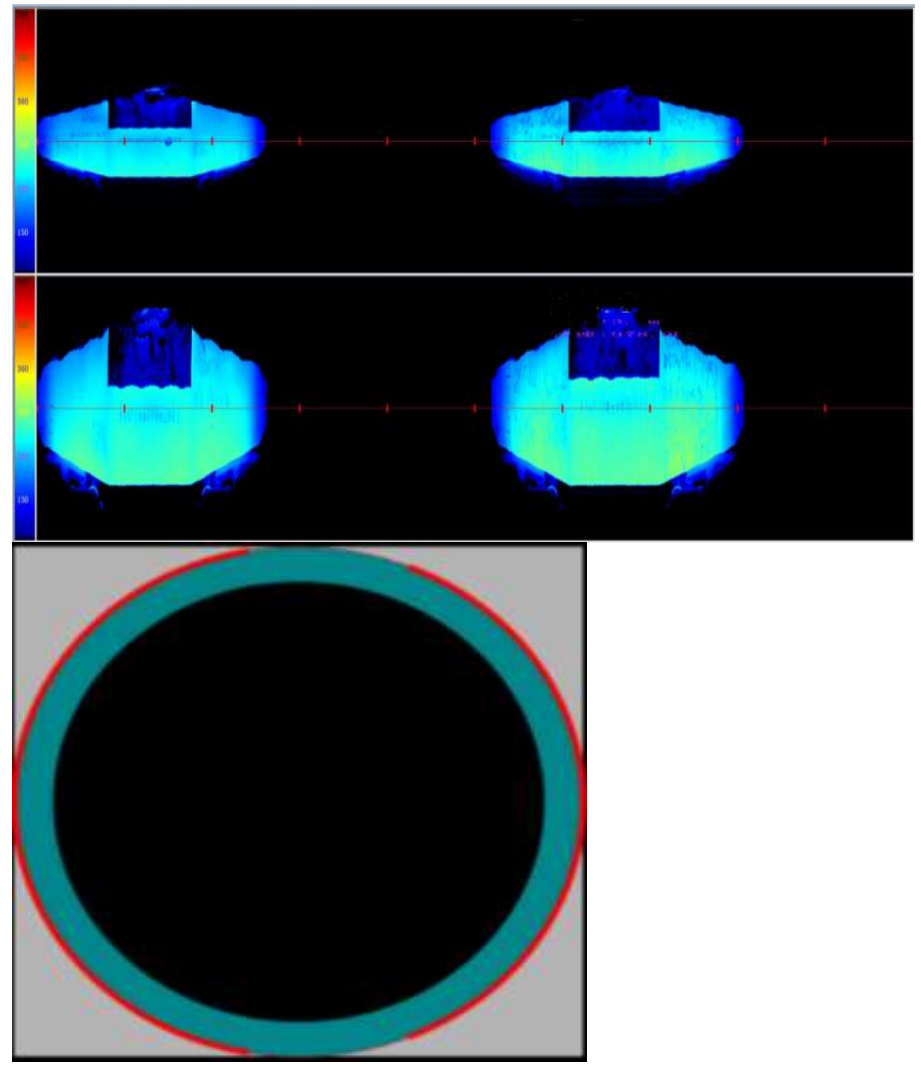

Figure 7. Thermal imaging and axial temperature profile.

As shown in Fig.7, the speed of torpedo car with two tanks is about $6-10 \mathrm{~km} / \mathrm{h}$, we use the pseudo-color to describe the surface temperature field distribution on the two sides of the torpedo car. By comparing the temperature corresponding to the color scale on the left, the temperature and relative height of each surface position can be visually distinguished from the figure. Through the temperature information fed back from the graph, it is analyzed and inferred whether the torpedo tank body lining is damaged, whether there is sediment at the bottom, etc., as well as the specific geometric dimensions and precise physical location on the surface of the torpedo car when the above situation occurs. Based on the heat transfer model and boundary conditions[12], we perform linear mapping, which can simulate the radial temperature distribution of the car shell, providing a basis for the design of refractory linings and the establishment of accurate heat transfer models.

\subsection{Statistical analysis of heat map}

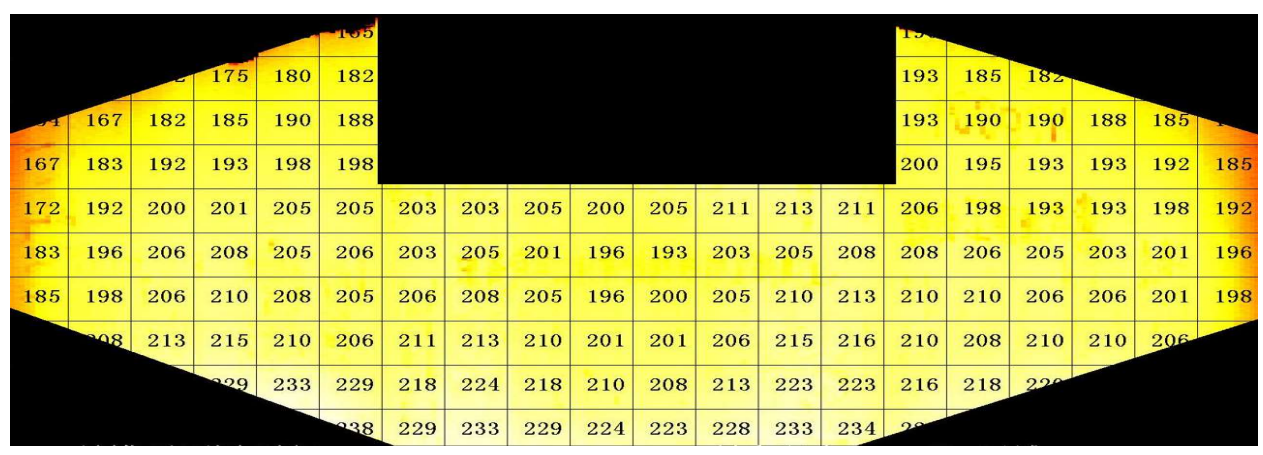

Figure 8. image reconstruction of torpedo car. 
As shown in Fig.8, image reconstruction and unit segmentation (20*10 elements) are performed based on the axial temperature data and the physical model of the shell.

Each unit is preset to a limit value of $350^{\circ} \mathrm{C}$. Whenever a temperature value higher than this preset value is read, the system outputs the high temperature alarm status, and displays the temperature value table of each unit on the monitor screen, and informs the operator of a "high temperature" alarm by voice.

\section{CONCLUSION}

The temperature of the torpedo car shell is 100-500 degrees Celsius. Low-cost area array thermal imaging cameras mainly use uncooled long-wave infrared technology (814 microns). There are phenomena such as large temperature measurement errors in hightemperature sections, inaccurate data, poor environmental adaptability[13, 14].According to Wien's law, to accurately measure the temperature, the mid-wave infrared imaging technology can obtain the best performance. The cooling medium wave infrared scanning temperature measurement system can cool the detector thermoelectricity and compensate the temperature, which can output data steadily day and night; the unique large field of view (0-180 degrees) is especially suitable for online detection of moving objects; in order to adapt to rain, snow, fog and other weather, the installation distance should not be too far; in addition, it can simulate the lining thickness and locate the fault accurately. To assist in the analysis and infer the damage trend of the inner lining, and then help determine when the torpedo car is repaired which not only greatly reduces the manpower and material costs, but also reduces the number of spare torpedo cars.

Conflicts of Interest: The authors declare no conflicts of interest.

\section{REFERENCES}

1. J. Y. Zhao, Y. Zang, Q. Qin, and D. P. Wu, "Thermo-Mechanical Analysis of Torpedo Car," Applied Mechanics \& Materials 44-47, 1198-1202 (2011).

2. V. Yemelyanov, T. Tochilkina, E. Vasilieva, A. Nedelkin, and E. Shved, "Computer Diagnostics Of The Torpedo Ladle Cars," in International Conference on Inventive Material Science Applications, V. Bindhu, H. H. Wang, and G. Ranganathan, eds. (Amer Inst Physics, Melville, 2018).

3. S. L. Jin, H. Harmuth, D. Gruber, A. Buhr, S. Sinnema, and L. Rebouillat, "Thermomechanical modelling of a torpedo car by considering working lining spalling," Ironmaking \& Steelmaking 47, 145-149 (2020).

4. A. Goldwaser and A. Schutt, "Optimal Torpedo Scheduling," J. Artif. Intell. Res. 63, 955-986 (2018).

5. R. Usamentiaga, P. Venegas, J. Guerediaga, L. Vega, J. Molleda, and F. G. Bulnes, "Infrared thermography for temperature measurement and non-destructive testing," Sensors (Basel) 14, 12305-12348 (2014).

6. S. Dai, H. Nie, W. Yi, Y. Yang, J. Li, and D. Li, "Research on a new method of multi-zone high temperature point tracking of rotary kiln surface temperature based on equal angle scanning," Infrared Physics \& Technology 103(2019).

7. R. A. Botelho and A. Calente, "Online monitoring of torpedo car shells," Proceedings of Spie the International Society for Optical Engineering (2001).

8. Z. Jiang, Y. Guo, D. Pan, W. Gui, and X. Maldague, "Polymorphic Measurement Method of FeO Content of Sinter Based on Heterogeneous Features of Infrared Thermal Images," IEEE Sensors Journal, 1-1 (2021).

9. G. C. Holst, R. Driggers, and O. Furxhi, "Design considerations for advanced MWIR target acquisition systems," Appl. Opt. 59(2020).

10. Bose, "Planck's law and light quantum hypothesis," Zeitschrift Fur Physik 26, 178-181 (1924).

11. P. Saurel, "On Wien's displacement law," Phys. Rev. 30, 356-358 (1910).

12. Y. Liu, Y. Xing, C. Yang, C. Li, and C. Xue, "Simulation of heat transfer in the progress of precision glass molding with a finite element method for chalcogenide glass," Appl. Opt. 58(2019).

13. G. A. Findlay and D. R. Cutten, "Comparison of performance of 3-5-and 8-12- $\mu$ m infrared systems," Appl. Opt. 28, 50295020 (1989).

14. T. Meitzler, G. Gerhart, E. Sohn, and P. Collins, Comparison of the performance of 3-5 and 8-12 micron infrared cameras, SPIE's International Symposium on Optical Engineering and Photonics in Aerospace Sensing (SPIE, 1994), Vol. 2224. 